\title{
Tarefas de Nomeação Seriada Rápida: Rastreando a Dificuldade de Leitura ${ }^{1}$
}

\author{
Cláudia Nascimento Guaraldo Justi ${ }^{2}$ \\ Universidade Federal de Juiz de Fora \\ Natália Cunha \\ Fundação Educacional Machado Sobrinho
}

\begin{abstract}
RESUMO - Este estudo analisou a precisão de medidas de nomeação seriada rápida (NSR) em identificar os estudantes em risco/sem risco de dificuldade de leitura. Tarefas de NSR requerem a nomeação rápida de diferentes estímulos visuais apresentados em um cartão. Duzentas e treze crianças realizaram as tarefas de NSR quando estavam na Educação Infantil e, dessas, 174 realizaram uma tarefa de leitura quando estavam no $1^{\circ}$ ano do Ensino Fundamental. Análises da curva ROC (Receiver Operating Characteristic) indicaram que as medidas de NSR (objetos, cores, números e letras) são razoáveis em identificar o risco/não risco de dificuldade de leitura (área sob a curva $\geq 0,70$ ), sendo a NSR de números a medida com melhores índices de sensibilidade (88\%) e especificidade (50\%).
\end{abstract}

Palavras-chave: nomeação seriada rápida, rastreamento, leitura, distúrbios da leitura

\section{Rapid Automatized Naming Tasks: Screening Reading Difficulty}

\begin{abstract}
The aim of this study was to analyze the rapid automatized naming (RAN)'s accuracy for identifying students at risk/not at risk for reading difficulty. Two hundred and thirteen children performed the RAN tasks when they were in the last year of Kindergarten and 174 among them, accomplished the reading task when they were in the first grade of Elementary School. Analyses based on the ROC curve (Receiver Operating Characteristic) indicated that all the RAN measures (objects, colors, numbers, and letters) had a fair accuracy for identifying students at risk/not at risk for reading difficulty (AUC $\geq 0.70$ ). RAN numbers had the best classification accuracy presenting sensitivity and specificity indexes of $88 \%$ and $50 \%$, respectively.
\end{abstract}

Keywords: rapid automatized naming, screening, reading, reading disabilities

O aprendizado da leitura representa um marco muito importante no desenvolvimento do ser humano. Uma nova forma de acesso à informação se torna disponível, o que amplia consideravelmente as possibilidades de desenvolvimento do indivíduo, seja no âmbito social, emocional, cognitivo ou cerebral. Sabe-se hoje que não saber ler e escrever é um fator de risco para o desenvolvimento de demências (Brucki, 2010) e que a doença de Alzheimer progride mais rapidamente entre as pessoas que não leem do que entre as pessoas que leem (Lee et al., 2008).

O termo leitura é utilizado na literatura científica, comumente, para se referir à precisão na decodificação de palavras escritas (transformação de um input ortográfico em um output fonológico ou semântico - precisão de leitura), à rapidez ou fluidez na decodificação (fluência de leitura), à construção do significado de textos escritos (compreensão de textos), podendo ser usado também para se referir, de forma geral, a todos esses aspectos. Subjacente ao desenvolvimento de cada componente da leitura (precisão, fluência e compreensão) está um conjunto de variáveis cognitivas e metalinguísticas, sendo algumas delas o conhecimento do nome das letras, a memória de trabalho, a consciência fonológica (habilidade de perceber

1 Artigo baseado em pesquisa financiada pelo $\mathrm{CNPq}$

2 Endereço completo para correspondência: Departamento de Psicologia, Instituto de Ciências Humanas, Universidade Federal de Juiz de Fora, Campus Universitário, Juiz de Fora, MG, Brasil, CEP: 36036-330.

E-mail: claudia.ngjusti@gmail.com e manipular os sons das palavras faladas; Bryant \& Bradley, 1987), a consciência morfológica (consciência da estrutura morfológica das palavras e habilidade de refletir sobre e manipular essa estrutura; Carlisle, 1995), o vocabulário e a habilidade de fazer inferências (Snowling \& Hulme, 2005). Essas variáveis não se relacionam igualmente com todos os componentes da leitura. Por exemplo, a habilidade de fazer inferências é particularmente importante para a compreensão de textos, enquanto a consciência fonológica é importante para a decodificação (ver Snowling \& Hulme, 2005 e Stone, Siliman, Ehren, \& Apel, 2004, para informações sobre como cada uma dessas variáveis se relaciona com os componentes da leitura). Em suma, a leitura é multifacetada e o conhecimento dessa complexidade é indispensável para a elaboração e a implementação de intervenções com foco no desenvolvimento da leitura (O'Connor \& Vadasy, 2011).

A complexidade da leitura vem sendo revelada por meio de pesquisas básicas, representando um sucesso na história da Psicologia (Johnston, Barnes, \& Desrochers, 2008). Muito do conhecimento gerado por essas pesquisas motivaram o surgimento de uma prática baseada em evidências com a finalidade de prevenir a dificuldade de leitura (O'Connor \& Vadasy, 2011; Snow, Burns, \& Griffin, 1998). Sabe-se hoje que a prevenção é muito mais efetiva do que a remediação da dificuldade de leitura (Torgesen, 1998).

Existem diferentes abordagens de prevenção (Macklen, 2013), sendo a Resposta à Intervenção (do inglês Response to Intervention $[\mathrm{RTI}]$ ) a mais utilizada em se tratando de 
prevenção de dificuldade de leitura. A RTI propõe uma intervenção em diferentes camadas, sendo a primeira voltada para o aprimoramento da instrução, tornando-a mais cientificamente embasada e de boa qualidade, beneficiando todas as crianças da escola. A segunda camada é voltada para os(as) estudantes que não mostraram os níveis de progresso esperados em relação a seus pares ou a normas nacionais após o $1^{\circ}$ passo (classificados como tendo risco de dificuldade de leitura), sendo a intervenção realizada em pequenos grupos, por tempo determinado. Já a terceira camada é voltada para os(as) estudantes que não apresentaram progressos após o $2^{\circ}$ passo, sendo a intervenção comumente realizada em grupos menores ou mesmo individualmente, por tempo indeterminado (Fletcher \& Vaughn, 2009). O conhecimento científico sobre o desenvolvimento da leitura é central para a intervenção realizada em todas as camadas.

O objetivo da RTI é garantir uma instrução de boa qualidade para todos(as) os(as) estudantes e a identificação (por meio da aplicação de tarefas de rastreamento), o mais cedo possível, de crianças que estão em risco de apresentarem dificuldade de leitura, para que essas possam receber mais atenção e serem mais intensamente e devidamente estimuladas. Dessa forma, um bom conhecimento sobre as variáveis preditivas do desenvolvimento da leitura é fundamental, uma vez que, entre outros fatores, são elas que potencialmente ajudarão a identificar as crianças que têm risco de apresentarem dificuldade de leitura. Além das variáveis já citadas previamente, uma variável que consistentemente é apontada como sendo altamente preditiva do desenvolvimento da leitura é a nomeação seriada rápida (Araújo, Reis, Petersson, \& Faísca, 2015; Georgiou, Parrila, Cui, \& Papadopoulo, 2014; Kirby, Pfeiffer, \& Parrila, 2003; Norton \& Wolf, 2012; Wolf, 2014). Tarefas de nomeação seriada rápida requerem que os indivíduos nomeiem, em voz alta, da forma mais correta e rápida quanto possível, um conjunto de estímulos visuais, dispostos em série em um cartão. Enquanto o indivíduo realiza a tarefa são registrados tanto o tempo decorrido entre a nomeação do primeiro ao último estímulo, bem como os erros cometidos durante esse processo.

Denckla e Rudel (1976) foram as primeiras a demonstrar que crianças com dificuldade na nomeação seriada rápida (i.e., com nomeação mais lenta dos estímulos) tendem a apresentar dificuldade de leitura. Essas pesquisadoras elaboraram as tarefas de Nomeação Automatizada Rápida (do inglês Rapid Automatized Naming [RAN]) que acabaram se tornando tarefas padrão para avaliar a nomeação seriada rápida. As tarefas desenvolvidas por Denckla e Rudel consistem, cada uma, de um cartão contendo 50 estímulos visuais, todos de alta frequência de ocorrência, dispostos, aleatoriamente, em 10 colunas e cinco linhas, cabendo ao(à) participante nomeá-los o mais rapidamente possível. Essas autoras elaboraram quatro tarefas que se diferenciam apenas pelo tipo de estímulos que contêm: objetos desenhados (tarefa de nomeação seriada rápida de objetos), quadrados coloridos (tarefa de nomeação seriada rápida de cores), números (tarefa de nomeação seriada rápida de números) e letras (tarefa de nomeação seriada rápida de letras).

As tarefas elaboradas por Denckla e Rudel (1976) são as mais comumente utilizadas pelos(as) pesquisadores(as), a fim de avaliar a nomeação seriada rápida. No entanto, outras tarefas também podem ser encontradas na literatura. Essas outras tarefas, apesar de muito semelhantes às de Denckla e Rudel, diferem dessas na quantidade de itens em uma mesma tarefa (15 itens em Landerl \& Willbuger, 2010; 25 itens em Zhou et al., 2014; 36 itens em Logan, Schatschneidere, \& Wagner, 2011; 40 itens em Shapiro, Carroll, \& Solity, 2013; 48 em Clarke, Hulme, \& Snowling, 2005) e/ou na quantidade de estímulos diferentes em cada tarefa (seis itens diferentes em Pauly, Linkersdörfer, \& Lindberg, 2011, e Logan et al., 2011; 10 a 25 itens diferentes em Clarke et al., 2005; 20 itens em Cobbold, Passenger, \& Terrel, 2003). Embora essas variações pudessem, a princípio, afetar a relação entre a nomeação seriada rápida e a leitura, a meta-análise realizada por Araújo et al. (2015) revelou que nenhum desses aspectos (variação no tamanho da tarefa ou na quantidade de itens diferentes incluídos) teve um impacto significativo na magnitude da correlação entre a nomeação seriada rápida e a leitura.

Apesar da exata natureza da relação entre a nomeação seriada rápida e o desenvolvimento da leitura ainda precisar ser estabelecida (ver Justi \& Roazzi, 2013, para uma revisão a esse respeito), atualmente, há muitas evidências de que o desempenho em tarefas de nomeação seriada rápida prediz o desempenho em tarefas de leitura (Araújo et al., 2015). Em muitos desses estudos (Kirby et al., 2003; Parrila, Kirby, \& McQuarrie, 2004; Schatschneider, Fletcher, Francis, Carlson, \& Foorman, 2004), as tarefas de nomeação seriada rápida foram administradas quando as crianças ainda estavam na Educação Infantil e as tarefas de leitura foram aplicadas quando as mesmas crianças se encontravam no Ensino Fundamental. Nesses estudos, comumente, os(as) pesquisadores(as) realizaram análises de regressão, a fim de avaliar o quanto o desempenho nas tarefas de nomeação seriada rápida prevê o desempenho nas tarefas de leitura.

Evidências de contribuições significativas da nomeação seriada rápida para a leitura têm sido apresentadas por estudos realizados com falantes de diferentes línguas, como, por exemplo, o inglês (Kruk \& Funk, 2014), o alemão (Wimmer, Mayringer, \& Landerl, 2000), o norueguês (Lervåg, Bråten, \& Hulme, 2009), o finlandês (Lepola, Poskiparta, Laakkonen, \& Nurmi, 2005), o holandês (Verhagen, Aarnoutse, \& van Leeuwede, 2008), o espanhol (López-Escribano \& Katzir, 2008), o francês (Plaza \& Cohen, 2004) e o português brasileiro (Justi \& Roazzi, 2012).

De forma geral, os estudos têm evidenciado que variações na nomeação seriada rápida continuam a explicar uma porcentagem significativa de variações na habilidade de leitura, mesmo após o controle de variáveis importantes, como a consciência fonológica (Albuquerque, 2012; Di Filippo, et al., 2005; Justi \& Roazzi, 2012), o conhecimento do nome das letras (Levarg et al., 2009), a inteligência, a memória de trabalho fonológica e a consciência morfológica (Justi \& Roazzi, 2012).

Araújo et al. (2015) realizaram uma meta-análise que incluiu 137 estudos que investigaram, empiricamente, a relação entre a nomeação seriada rápida e a leitura. Os resultados revelaram uma correlação mais forte entre a nomeação seriada rápida e a leitura de palavras ou de um texto do que entre a nomeação seriada rápida e a leitura de 
pseudopalavras (estímulos que seguem as regras fonotáticas e ortográficas da língua, mas não têm nenhum significado associado a eles, por exemplo, 'zato') ou a compreensão de texto, apesar de todas as correlações terem sido significativas.

Os resultados da meta-análise de Araújo et al. (2015) também evidenciaram que, independentemente da língua considerada nos estudos, a correlação da nomeação seriada rápida com a fluência de leitura é mais forte do que a correlação da nomeação seriada rápida com a precisão de leitura. Também foi observado que o ano escolar do(a) participante não afeta significativamente a correlação entre a nomeação seriada rápida e a leitura quando a fluência de leitura é considerada, mas afeta quando a precisão de leitura é considerada. Nesse último caso, foi encontrado que a correlação aumenta da Educação Infantil para o $1^{\circ}$ e $2^{\circ}$ anos e diminui nos anos consecutivos, à medida que os(as) estudantes se tornam leitores(as) mais proficientes.

Araújo et al. (2015) encontraram, também, que independentemente do ano escolar das crianças, todas as quatro medidas de nomeação seriada rápida (cores, números, objetos e letras) se correlacionam significativamente com a leitura. No entanto, o desempenho em tarefas alfanuméricas é mais fortemente correlacionado com a leitura $(r=51$ para letras e $r=48$ para números) do que o desempenho em tarefas não alfanuméricas ( $r=35$ para objetos e $r=33$ para cores).

De acordo com Jenkins, Hudson e Johnson (2007) e Ritchey e Speece (2004), estudos que avaliam o quanto as variações em uma variável preveem as variações em outra variável, como os citados anteriormente, são úteis para identificar medidas que têm o potencial para rastrear possíveis dificuldades no desenvolvimento da leitura. No entanto, segundo esses autores, a análise da precisão de classificação do risco/não risco de dificuldade de leitura dessas medidas é fundamental, pois explicita o grau de eficiência das mesmas em identificar o risco/não risco individual.

Tendo como base a curva ROC (Receiver Operating Characteristic), a precisão de classificação de uma medida é descrita pela área sob a curva e pelos índices de sensibilidade e especificidade. A área sob a curva é um índice da precisão com que uma medida classifica os indivíduos em dois grupos, no caso do presente trabalho, com dificuldade de leitura e sem dificuldade de leitura. A sensibilidade de uma medida de rastreamento da dificuldade de leitura é a probabilidade de a mesma identificar como tendo risco de dificuldade de leitura as pessoas que realmente terão dificuldade de leitura. Já a especificidade é a probabilidade de a medida identificar como não tendo risco de dificuldade de leitura as pessoas que realmente não terão dificuldade de leitura. Nesse sentido, a sensibilidade e a especificidade são propriedades das medidas. A medida ideal, com $100 \%$ de sensibilidade e $100 \%$ de especificidade, raramente existe na prática, pois a tentativa de melhorar a sensibilidade frequentemente tem o efeito de diminuir a especificidade. A recomendação é que uma medida de rastreamento para ser considerada boa tenha uma sensibilidade de $90 \%$ ou mais (Jenkins et al., 2007), associada a uma especificidade de, no mínimo, 50\% (Catts, Petscher, Schatschneider, Bridges, \& Mendoza, 2009) ou 80\% (Compton et al., 2010).

Com o objetivo de identificar estudos que avaliaram a precisão de classificação do risco/não risco de dificuldade de leitura das medidas de nomeação seriada rápida, uma busca nas bases de dados Scielo e BVS-Psi foi realizada em julho de 2016. Nessa busca, os descritores nomeação seriada rápida ou nomeação automatizada rápida foram utilizados e nenhum estudo foi encontrado. Com o mesmo objetivo, nesse mesmo mês e ano, foi realizada uma busca na base de dados PsycInfo, usando os descritores rapid naming e screening ou specificity and sensitivity, tendo sido encontrado apenas um estudo: o de Bishop e League (2006).

Bishop e League (2006) analisaram a sensibilidade e a especificidade da nomeação seriada rápida na classificação do risco/não risco de dificuldade de leitura (uma medida de fluência de leitura foi usada como critério), tendo como amostra 79 crianças falantes do inglês, matriculadas, inicialmente, na Educação Infantil. As crianças foram avaliadas em sua habilidade de leitura anualmente, do $1^{\circ}$ ao $4^{\circ}$ ano do Ensino Fundamental. Tarefas de nomeação seriada rápida de objetos e de cores foram administradas no início e no meio do último ano da Educação Infantil. Os resultados revelaram que a nomeação seriada rápida (de cores e objetos), quando avaliada no início do último ano da Educação Infantil, apresentou índices de sensibilidade variando de $57 \%$ a $75 \%$ e índices de especificidade variando de $77 \%$ a $89 \%$. Ao ser avaliada no meio do último ano da Educação Infantil, a nomeação seriada rápida apresentou valores mais altos de sensibilidade (de $89 \%$ a $99 \%$ ), associados a valores mais baixos de especificidade (de $48 \%$ a $82 \%$ ).

Atualmente, muitos estudos têm avaliado a precisão de classificação de baterias de rastreamento, sendo a nomeação seriada rápida uma das tarefas que as compõem (Catts, Nielsen, Bridges, Liu, \& Bontempo, 2015; Compton, Fuchs, Fuchs, \& Bryant, 2006; Compton et al., 2010; Puolakanaho et al., 2007; Thompson, Hulme, Nash, Gooch, HayiouThomas \& Snowling, 2015). Apesar de ser fundamental a análise de qual combinação de preditores (bateria) é melhor para detectar o risco/não risco de dificuldade de leitura, parece importante que primeiro a precisão de classificação de cada medida seja aferida para, em seguida, verificar qual combinação de preditores pode aumentar a eficiência na classificação do risco/não risco de dificuldade de leitura.

A partir dos estudos que evidenciam a importância de se identificar, o mais cedo possível, crianças com risco de dificuldade de leitura (Fletcher \& Vaughn, 2009) e daqueles que mostram o potencial das medidas de nomeação seriada rápida para serem usadas para esse propósito (Araújo et al., 2015), o presente estudo foi proposto. O principal objetivo foi verificar a precisão de classificação do risco/ não risco de dificuldade de leitura das medidas de nomeação seriada rápida. Além disso, a fidedignidade das medidas de nomeação seriada rápida também foi avaliada, uma vez que não foi encontrado nenhum estudo com crianças falantes do português brasileiro que tenha efetuado tal análise.

O presente estudo se justifica pelo grande volume de pesquisas que vêm evidenciando o poder das medidas de nomeação seriada rápida na predição do desenvolvimento da leitura e pela escassez de estudos analisando a precisão de classificação do risco/não risco de dificuldade de leitura dessas medidas separadamente. Nesse sentido, cabe ressaltar que, na busca efetuada, foi encontrado apenas um estudo 
com falantes do inglês (Bishop \& League, 2006) e nenhum estudo com falantes do português brasileiro.

Como mencionado por Torgesen (1998), "a melhor solução para o fracasso no aprendizado da leitura é alocar recursos para a identificação precoce e a prevenção" (p. 32). Dessa forma, o presente estudo tenta contribuir investigando uma das medidas com potencial significativo de ser eficiente na identificação de crianças com risco/sem risco de apresentarem dificuldade de leitura. Os resultados desse estudo poderão ser usados para uma prática baseada em evidências nas escolas brasileiras.

No presente estudo, as tarefas de nomeação seriada rápida utilizadas foram adaptadas das tarefas elaboradas por Denckla e Rudel (1976; Wolf \& Denckla, 2005). Essa escolha se justifica porque, como mencionado, essas tarefas são as mais frequentemente utilizadas pelos(as) pesquisadores(as) e porque o estudo de Araújo et al. (2015) apresentou evidências de que a relação entre a nomeação seriada rápida e a leitura não é afetada pelo formato da tarefa de nomeação seriada rápida utilizada, tornando a escolha de quais tarefas utilizar mais fácil.

\section{Método}

\section{Participantes}

Este estudo, de delineamento longitudinal, contou com a participação de 213 crianças matriculadas, inicialmente, no $2^{\circ}$ período da Educação Infantil. Dessas 213 crianças, 99 estavam matriculadas em escolas particulares, sendo 47 do sexo masculino e 52 do sexo feminino; 114 estavam matriculadas em escolas públicas, sendo 56 do sexo masculino e 58 do sexo feminino. Considerando toda a amostra, a idade média das crianças no início do estudo foi de, aproximadamente, 6 anos, com um desvio padrão de 3,7 meses. Nove escolas particulares e sete escolas públicas participaram desta pesquisa, sendo essas escolas de diferentes regiões (sul, norte, leste e centro) da cidade de Juiz de Fora, Minas Gerais. Não foram incluídas na amostra final as crianças com algum diagnóstico de deficiência intelectual, como, por exemplo, Síndrome de Down e Síndrome do $\mathrm{X}$-Frágil ou que apresentavam deficiências sensoriais, como a cegueira ou a surdez.

Este estudo é parte de um estudo maior, intitulado Preditores do desenvolvimento da leitura e da escrita: Um estudo longitudinal, cuja realização foi aprovada pelo Comitê de Ética em Pesquisa com seres humanos da Universidade Federal de Juiz de Fora (Protocolo: 16525513.9.0000.5147). Apenas as crianças cujos pais assinaram o Termo de Consentimento Livre e Esclarecido participaram da pesquisa.

\section{Instrumentos}

Nomeação seriada rápida (adaptado de Wolf \& Denckla, 2005). Quatro tarefas de nomeação seriada rápida foram utilizadas: tarefa de nomeação seriada rápida de objetos, de cores, de números e de letras. Cada tarefa é composta por um cartão (tamanho de duas folhas A4, quando aberto) contendo cinco tipos diferentes de itens, conforme o tipo de tarefa: cinco cores diferentes, cinco letras diferentes, cinco números diferentes e cinco objetos diferentes. Os cinco tipos de itens de cada tarefa são apresentados em ordem randômica e com repetição em 10 colunas e cinco linhas, totalizando 50 itens por cartão. $\mathrm{Na}$ parte da frente de cada cartão, quando o mesmo está fechado, há 10 itens distribuídos em ordem randômica e com repetição (cada item aparece duas vezes) em cinco colunas e duas linhas. A parte da frente do cartão é usada na fase de treinamento para verificar se o(a) participante conhece o nome dos cinco tipos de itens presentes na tarefa e para instruí-lo quanto à realização da mesma. Nessa tarefa, são registrados, durante a fase de testagem, o tempo que o(a) participante leva para nomear todos os itens presentes em cada cartão e o número de erros cometidos.

Leitura. A tarefa TPL1 - Teste de Precisão de Leitura - $1^{\circ}$ ano foi utilizada no presente estudo para avaliar a precisão de leitura de palavras. Essa tarefa, desenvolvida pelo Grupo de Pesquisa em Cognição e Linguagem (CogLin - UFJF), conta com 80 palavras apropriadas para crianças do 10 ano do Ensino Fundamental (de acordo com o trabalho de Pinheiro, 1996). Nessa tarefa, todas as palavras (balanceadas quanto à frequência de ocorrência e regularidade) estão impressas na cor preta, em fonte Times New Roman, número 12, em um cartão branco (tamanho A4). As 80 palavras estão distribuídas em oito colunas contendo 10 palavras cada. O escore de cada criança nessa tarefa consiste no número de palavras lidas em voz alta corretamente. Análises realizadas pelo grupo CogLin revelaram que essa medida tem uma fidedignidade de consistência interna de 0,99 (estatística Kuder-Richarson).

\section{Procedimento}

Adaptação das tarefas de nomeação seriada rápida. Todas as tarefas de nomeação seriada rápida administradas no presente estudo foram adaptadas de Wolf e Denckla (2005), a fim de torná-las adequadas à amostra. Com base no trabalho de Pinheiro (1996), as cinco letras mais frequentes do português brasileiro (o, a, s, e, r) foram selecionadas para compor a tarefa de nomeação seriada rápida de letras. As letras, em formato minúsculo, foram impressas em fonte Arial, tamanho 55, na cor preta. Os cinco números utilizados na tarefa de nomeação seriada rápida de números foram escolhidos entre os 10 números de um único dígito que eram dissílabos $(4,7,8,9,5)$. Os números foram impressos em fonte Arial, tamanho 55, na cor preta. Os cinco objetos utilizados na tarefa de nomeação seriada rápida de objetos (gato, faca, olho, bola, uva) são dissílabos e estão entre os cinco itens mais típicos de suas respectivas categorias, de acordo com o trabalho de Pinheiro (2007). Os objetos desenhados foram impressos na cor preta. Por fim, as cinco cores com os mais elevados índices de tipicidade (preto, vermelho, azul, verde, amarelo), com base no trabalho de Pinheiro (2007), foram selecionadas para compor a tarefa de nomeação seriada rápida de cores. Quadrados coloridos com cada uma dessas cinco cores foram impressos, compondo essa tarefa. 
Aplicação das tarefas de nomeação seriada rápida. Cada criança foi avaliada individualmente em um ambiente dentro da própria escola, sendo necessários, aproximadamente, 10 minutos para a realização das quatro tarefas. Todas as tarefas de nomeação seriada rápida foram aplicadas da mesma forma e pelos mesmos(as) pesquisadores(as), em duas sessões diferentes, sendo a primeira denominada teste (efetuada quando as crianças estavam no final do $2^{\circ}$ período da Educação Infantil) e a segunda, realizada uma semana após a primeira, denominada reteste. Em cada uma das sessões, a primeira tarefa apresentada à criança foi a de nomeação seriada rápida de objetos, seguida, sucessivamente, da de cores, da de números e da de letras. Em cada sessão, a aplicação de cada tarefa de nomeação seriada rápida se iniciou com a fase de treinamento, seguida da fase de testagem.

Fase de treinamento. O cartão era colocado fechado sobre a mesa, em frente à criança, e solicitava-se que ela dissesse o nome de cada item presente na parte frontal do mesmo. Caso a criança não soubesse (não dissesse ou errasse) o nome de algum item, a aplicação da tarefa não era continuada (isso foi registrado no banco de dados como dados faltantes) e apresentava-se a ela a próxima tarefa, de acordo com a ordem mencionada anteriormente. Esse procedimento se justifica porque as quatro tarefas são independentes, logo uma criança pode não saber o nome de um objeto, mas pode saber o nome dos itens presentes nas demais tarefas de nomeação seriada rápida. Caso a criança acertasse o nome de todos os itens presentes na parte frontal do cartão, ela recebia a instrução de que o próximo passo era tentar dizer o nome de todos os itens (10) da parte frontal do cartão, sendo o primeiro item a ser nomeado apontado pelo(a) pesquisador(a), bem como a direção da leitura (da esquerda para a direita e de cima para baixo) demonstrada manualmente. Foi enfatizado que a criança não deveria parar entre as linhas e que deveria tentar nomear da forma mais rápida e correta quanto possível. A criança também foi instruída de que, caso cometesse algum erro, poderia corrigi-lo, dizendo o nome correto do item o mais rapidamente possível. Foi dito à criança que esperasse o(a) pesquisador(a) dizer "pode começar" para iniciar a nomeação dos itens. Após a nomeação dos itens, $o$ (a) pesquisador(a) avaliava se a criança tinha seguido todas as instruções corretamente e, caso ela não tivesse seguido as instruções, o procedimento era repetido.

Fase de testagem. Tendo se certificado de que a criança entendeu as instruções para a realização das tarefas de nomeação seriada rápida, $\mathrm{o}$ (a) pesquisador(a) abria o cartão contendo os 50 itens e repetia a instrução de que todos os itens deveriam ser nomeados, sem interrupção, da forma mais rápida e correta quanto possível. Ao dizer "pode começar", o(a) pesquisador(a) acionava o cronômetro imediatamente e acompanhava o desempenho da criança em uma folha de respostas (reprodução, em miniatura, das quatro tarefas). Os erros cometidos durante o processo eram registrados nessa folha, bem como o tempo (medido em segundos) que a criança levava para nomear do primeiro ao último item. Autocorreções foram registradas com a marcação AC sobre o item na folha de respostas e não foram contabilizadas como erros. Qualquer troca, omissão ou inversão foi considerada erro e registrada na folha de respostas com um X sobre o item omitido, nomeado erroneamente ou invertido. O desempenho das crianças nessas tarefas foi gravado para posterior checagem da precisão na aplicação e correção. Seis estudantes de psicologia aplicaram as tarefas.

Aplicação do TPL1 (Teste de Precisão de Leitura - $\mathbf{1}^{\circ}$ ano). Cada criança foi avaliada individualmente em um ambiente dentro da própria escola, sendo necessários, aproximadamente, 20 minutos para a realização dessa tarefa. A aplicação da tarefa de leitura ocorreu na $3^{\mathrm{a}}$ sessão, realizada oito meses após a aplicação das tarefas de nomeação seriada rápida na $1^{\mathrm{a}}$ sessão, com as crianças já cursando o $1^{\circ}$ ano do Ensino Fundamental. Há duas versões dessa tarefa: uma contendo as palavras impressas em letras maiúsculas e outra contendo as palavras impressas em letras minúsculas. As crianças puderam escolher em qual versão queriam ler as palavras. Após definir a versão, o cartão contendo as 80 palavras era colocado sobre a mesa, na frente da criança, e ela era orientada a ler as palavras em voz alta da melhor forma possível. Enquanto a criança lia as palavras, o(a) pesquisador(a) registrava o desempenho da criança em sua folha de resposta. Silabações foram consideradas erros apenas nos casos em que resultavam em regularizações de pronúncias (palavra 'gota' lida com 'o' aberto). Autocorreções não foram consideradas erros. Essa tarefa também foi gravada para possibilitar posterior checagem da precisão na aplicação e correção da tarefa. Seis estudantes de psicologia aplicaram as tarefas.

\section{Resultados}

Das 213 crianças que participaram da pesquisa, 201 conseguiram fazer a tarefa de nomeação seriada rápida de objetos; 195, a de cores; 165, a de números; e 103, a de letras. Esse dado evidencia uma dificuldade maior das crianças dessa amostra em conhecer o nome de estímulos alfanuméricos, principalmente, letras.

A fim de contemplar tanto a rapidez quanto a precisão na realização das tarefas de nomeação seriada rápida, optou-se por calcular o número de itens corretamente nomeados por segundo. Esse cálculo se deu a partir da aplicação da seguinte fórmula: (50-NSR_E) / NSR_T, onde 50 é igual ao número máximo de itens no cartão, NSR_E é igual ao número de erros cometidos na nomeação dos itens e NSR_T é igual ao tempo (em segundos) que a criança levou para dizer do primeiro ao último item. Em todas as análises realizadas nesse estudo, foram essas as variáveis consideradas.

A distribuição de todas as variáveis foi verificada pelo teste Kolmogorof-Smirnov. Os resultados evidenciaram que todas as quatro medidas de nomeação seriada rápida apresentaram distribuição normal (Nomeação Seriada Rápida de Objetos [NSRO]: $Z=0,60 ; p=0,864$; Nomeação Seriada Rápida de Cores [NSRC]: $Z=0,61 ; p=0,849$; Nomeação Seriada Rápida de Números [NSRN]: $Z=0,55 ; p=0,924$; Nomeação Seriada Rápida de Letras [NSRL]: $Z=0,88 ; p$ $=0,423)$. A medida de leitura apresentou uma distribuição positivamente assimétrica $(Z=2,8 ; p=0,000)$. Tendo em vista a distribuição da medida de leitura, bem como os propósitos deste estudo, essa variável foi dicotomizada: as crianças que apresentaram escores na medida de precisão 
de leitura um desvio-padrão $(27,15)$ abaixo da média da amostra $(29,22)$ foram classificadas como tendo dificuldade de leitura e as demais crianças foram classificadas como não tendo dificuldade de leitura. Das 174 crianças que realizaram a tarefa de precisão de leitura, 63 crianças $(36,8 \%)$ foram classificadas como tendo dificuldade de leitura e 113 foram classificadas como não tendo. Essa variável dicotomizada, denominada dificuldade de leitura, foi utilizada em todas as análises que requeriam uma medida de leitura.

A fim de verificar a fidedignidade das medidas de nomeação seriada rápida foi empregada a técnica teste-reteste e calculados os coeficientes de correlação entre os escores obtidos nas duas aplicações das medidas. Os resultados evidenciaram que os escores obtidos na primeira aplicação das medidas de nomeação seriada rápida correlacionaramse fortemente com os escores obtidos na segunda aplicação das mesmas medidas (NSRO: $r=0,71, p<0,001$; NSRC: $r=0,88, p<0,001$; NSRN: $r=0,89, p<0,001$; NSRL: $r=$ $0,82, p<0,001)$.

Tendo como objetivo verificar a precisão de classificação do risco/não risco da dificuldade de leitura de cada medida de nomeação seriada rápida, foram construídas curvas ROC com base nas classificações obtidas na variável dificuldade de leitura. A Tabela 1 apresenta os valores da área sob a curva, bem como os pontos de corte, a sensibilidade e a especificidade de todas as medidas de nomeação seriada rápida. Optou-se, neste estudo, por considerar três pontos

Tabela 1. Área sob a curva, pontos de corte, sensibilidade e especificidade das quatro medidas de nomeação seriada rápida

Classificação do Risco /

Não Risco da Dificuldade de Leitura

\begin{tabular}{|c|c|c|c|c|}
\hline \multirow{2}{*}{ Medidas } & \multicolumn{4}{|c|}{ Não Risco da Dificuldade de Leitura } \\
\hline & ASC (IC de 95\%) & PC & $\begin{array}{c}\text { Sensib. } \\
(\%)\end{array}$ & $\begin{array}{c}\text { Espec. } \\
(\%)\end{array}$ \\
\hline \multirow{3}{*}{ NSRO } & \multirow{3}{*}{$0,72(0,64-0,81)$} & 0,92 & 90 & 30 \\
\hline & & 0,81 & 76 & 51 \\
\hline & & 0,65 & 55 & 81 \\
\hline \multirow{3}{*}{ NSRC } & \multirow{3}{*}{$0,76(0,68-0,84)$} & 0,76 & 91 & 36 \\
\hline & & 0,71 & 77 & 51 \\
\hline & & 0,59 & 68 & 81 \\
\hline \multirow{3}{*}{ NSRN } & \multirow{3}{*}{$0,78(0,69-0,87)$} & 0,88 & 91 & 41 \\
\hline & & 0,81 & 88 & 50 \\
\hline & & 0,61 & 67 & 81 \\
\hline \multirow{3}{*}{ NSRL } & \multirow{3}{*}{$0,70(0,43-0,97)$} & 0,91 & 100 & 22 \\
\hline & & 0,76 & 60 & 51 \\
\hline & & 0,56 & 60 & 80 \\
\hline
\end{tabular}

Nota. NSRO = Nomeação seriada rápida de objetos; NSRC = Nomeação seriada rápida de cores; $\mathrm{NSRN}$ = Nomeação seriada rápida de números; $\mathrm{NSRL}=$ Nomeação seriada rápida de letras; $\mathrm{ASC}=$ Área sob a Curva; $\mathrm{IC}$ $=$ Intervalo de Confiança; $\mathrm{PC}=$ Ponto de corte; Sensib. $=$ sensibilidade; Espec. $=$ especificidade

de corte para cada medida, um mantendo a sensibilidade próxima a $90 \%$, outro mantendo a especificidade próxima a $50 \%$ e outro mantendo a especificidade próxima a $80 \%$.

Como pode ser observado na Tabela 1 , todas as medidas apresentaram uma área sob a curva variando de 0,70 a 0,78 , indicando que essas medidas discriminam razoavelmente dois grupos (com dificuldade de leitura e sem dificuldade de leitura). Entre as quatro medidas de nomeação seriada rápida, a de números foi a que apresentou a melhor área sob a curva $(0,78)$, bem como o melhor índice de sensibilidade (88\%) associado a um mínimo de 50\% de especificidade.

\section{Discussão}

Tendo em vista a importância de se identificar medidas adequadas para o rastreamento do risco/não risco de dificuldade de leitura de acordo com uma perspectiva preventiva, o presente estudo foi proposto. O principal objetivo foi verificar a precisão de classificação do risco/ não risco de dificuldade de leitura das medidas de nomeação seriada rápida.

Primeiramente, foi verificada a fidedignidade das medidas de nomeação seriada rápida, uma vez que as tarefas utilizadas foram adaptadas e não foi encontrado nenhum estudo a esse respeito realizado com crianças brasileiras. Tendo em vista que são medidas utilizadas para rastreamento de dificuldade de leitura, as evidências atestaram que as quatro medidas apresentaram boa fidedignidade (correlações acima de 0,70 , de acordo com Hogan, 2006), o que é consistente com o que foi encontrado por Wolf e Denckla (2005). Essas pesquisadoras também utilizaram a técnica teste-reteste para avaliar a fidedignidade das medidas de nomeação seriada rápida. Dados de 85 crianças estadunidenses, com idades entre 5 e 10 anos, fizeram parte dessas análises. Os resultados revelaram que a medida de nomeação de números foi a que apresentou o maior coeficiente de correlação $(r=0,89) \mathrm{e}$ a medida de nomeação seriada rápida de objetos foi a que apresentou o menor coeficiente de correlação $(r=0,81)$. Esse mesmo padrão foi encontrado no presente estudo.

Após a verificação da fidedignidade das medidas de nomeação seriada rápida, o uso da curva ROC possibilitou a avaliação da precisão de classificação do risco/não risco de dificuldade de leitura dessas mesmas medidas. As áreas sob a curva relativas a cada uma das medidas de nomeação seriada rápida evidenciaram que as mesmas são razoáveis para identificar o risco/não risco da dificuldade de leitura, pois foram encontrados valores entre 0,70 e 0,78 . Das quatro medidas de nomeação seriada rápida, a que apresentou melhores índices foi a de números. Considerando a área sob a curva $(0,78)$, bem como a sensibilidade $(88 \%)$ e a especificidade $(50 \%)$ associadas ao ponto de corte 0,81 , pode-se dizer que a nomeação seriada rápida de números ficou muito próxima de poder ser considerada uma boa medida de rastreamento, segundo o critério de Catts et al. (2009). Esse resultado é parcialmente consistente com o que foi encontrado por Araújo et al. (2015) em sua meta-análise, na qual a nomeação seriada rápida de números e de letras correlacionaram-se mais fortemente com a leitura do que a nomeação seriada rápida de cores e objetos.

Uma possível explicação do porquê, no presente estudo, a nomeação seriada rápida de letras apresentou o valor mais baixo de área sob a curva, bem como os piores índices de sensibilidade e especificidade, pode se assentar no fato de a mesma ter sido realizada por um número menor de 
crianças (aproximadamente, apenas a metade das crianças que fizeram as tarefas de cores e objetos conseguiram fazer a tarefa de letras). Essa redução amostral, consequência do não conhecimento por parte de muitas crianças do nome das cinco letras presentes na tarefa, pode ter criado um viés, de modo que as crianças que realizaram a tarefa foram aquelas propensas a não terem dificuldade de leitura. A fim de verificar essa hipótese ad hoc, foi criada uma variável dicotômica, representando o fato de a criança ter feito ou não a tarefa de nomeação seriada rápida de letras. Ao considerar essa nova variável, juntamente com a variável dificuldade de leitura, ficou evidenciado que, das 103 crianças que conseguiram fazer a tarefa de nomeação seriada rápida de letras, $94 \%$ foram classificadas como não tendo dificuldade de leitura. Ao mesmo tempo, das crianças que não conseguiram fazer a tarefa, $67 \%$ foram classificadas como tendo dificuldade de leitura. Esses resultados parecem dar suporte à hipótese ad hoc levantada no presente estudo.

Dos vários estudos encontrados na literatura sobre a relação entre a nomeação seriada rápida e a leitura, apenas o de Bishop e League (2006) investigou a precisão de classificação do risco/não risco de dificuldade de leitura das medidas de nomeação seriada rápida. Apesar dessas pesquisadoras terem colapsado as duas medidas de nomeação seriada rápida (objeto e cores) em seu estudo, o que prejudica comparações mais diretas com o presente estudo, é importante mencionar que, quando as tarefas de nomeação seriada rápida foram aplicadas no meio do último ano da Educação Infantil e a fluência de leitura avaliada no final da $1^{a}$ série, a nomeação seriada rápida apresentou uma sensibilidade de $82 \%$ associada a uma especificidade de $91 \%$. Como as pesquisadoras não relataram a sensibilidade e a especificidade associadas a outros pontos de corte, não é possível sabermos qual seria a especificidade, caso a sensibilidade tivesse sido fixada em, no mínimo, 90\%, como recomenda Jenkins et al. (2007). De qualquer forma, ao que tudo indica, seria um valor superior aos encontrados no presente estudo. Uma razão para essa inconsistência, possivelmente, é a habilidade de leitura considerada nas análises dos dois estudos. Bishop e League consideraram a fluência de leitura em seu estudo, enquanto no presente estudo a precisão de leitura é que foi considerada. Segundo a meta-análise de Araújo et al. (2015), a nomeação seriada rápida correlaciona-se mais fortemente com a fluência de leitura do que com a precisão de leitura.

Algumas ponderações são importantes de serem feitas. Uma se refere ao fato de as crianças que participaram deste estudo estarem ainda na Educação Infantil. Isso é positivo, tendo em vista que o objetivo de uma intervenção preventiva é a identificação, o mais cedo possível, de crianças com riscos/sem riscos de apresentarem dificuldade de leitura para que a intervenção ocorra, também, o mais cedo possível. No entanto, sabe-se que a relação das variáveis preditivas com a leitura varia ao longo do desenvolvimento. Por exemplo, Araújo et al. (2015) relataram que a força da correlação entre a nomeação seriada rápida e a leitura aumenta da Educação Infantil para o $1^{\circ}$ ano e do $1^{\circ}$ ano para o $2^{\circ}$ ano. Assim sendo, é importante que estudos futuros investiguem essa mesma questão em outras etapas do desenvolvimento, preferencialmente, por meio de um estudo longitudinal em que haja a aplicação de medidas de nomeação seriada rápida e de leitura a cada novo ano escolar.

Também a título de sugestão para trabalhos futuros, é importante que sejam incluídas medidas de outros componentes de leitura, como a fluência de leitura, por exemplo. No presente estudo optou-se por não incluir uma medida de fluência de leitura pelo fato das crianças brasileiras no $1^{\circ}$ ano do Ensino Fundamental ainda estarem iniciando o aprendizado da decodificação (muitas não conseguiram ler uma palavra). No entanto, é possível que a partir do $2^{\circ}$ ano do Ensino Fundamental uma medida de fluência de leitura já seja válida.

A falta de um padrão ouro para estabelecer a dificuldade no desenvolvimento da leitura em crianças brasileiras no $1^{\circ}$ ano do Ensino Fundamental constitui uma limitação do presente estudo. Os testes padronizados de leitura disponíveis foram desenvolvidos para crianças a partir do $2^{\circ}$ ano do Ensino Fundamental (antiga $1^{\text {a }}$ série). Dessa forma, foi necessário o desenvolvimento da tarefa experimental de leitura TPL1 para que este estudo pudesse ser realizado.

Ao investigar a precisão de classificação do risco/não risco de dificuldade de leitura das medidas de nomeação seriada rápida, este estudo contribuiu trazendo evidências de que essas medidas são razoáveis na classificação do risco/não risco de dificuldade de leitura, sendo a nomeação seriada rápida de números a melhor medida para ser usada na identificação do risco/não risco de dificuldade de leitura em amostras como a do presente estudo. Trata-se de uma tarefa de fácil aplicação e de rápida realização, características importantes para uma tarefa de rastreamento, tendo em vista que devem ser aplicadas em um número grande de crianças.

Para que a prevenção da dificuldade de leitura efetivamente faça parte da realidade das escolas brasileiras, alguns passos precisam ser dados. O primeiro é garantir um maior conhecimento dos estudos científicos sobre o desenvolvimento da leitura por parte dos profissionais que atuam diretamente na área educacional (psicólogos(as), professores(as), coordenadores(as), etc.). Saber quais habilidades deverão ser estimuladas na Educação Infantil visando à facilitação do aprendizado da leitura depende de um bom conhecimento da ciência da leitura. O segundo é a verificação da medida ou da combinação de medidas que mais precisamente identifica as crianças como estando ou não estando em risco de apresentarem dificuldade de leitura. Somente com medidas de rastreamento eficientes é que as crianças com risco potencial de apresentarem dificuldade de leitura poderão ser identificadas e, a partir daí, ter as suas necessidades devidamente avaliadas e atendidas. Dessa forma, espera-se que mais estudos possam investigar a precisão de classificação das diferentes medidas que reconhecidamente predizem, em nível geral, o desenvolvimento da leitura para que possamos, cada vez mais, ter uma prática baseada em evidências.

\section{Referências}

Albuquerque, C. (2012). Rapid naming contributions to reading and writing acquisition of European Portuguese. Reading and Writing, 25, 775-797. 
Araújo, S., Reis, A., Petersson, K., \& Faísca, L. (2015). Rapid automatized naming and reading performance: A meta-analysis. Journal of Educational Psychology, 107(3), 868-883.

Bishop, A., \& League, M. (2006). Identifying a multivariate screening model to predict reading difficulties at the onset of kindergarten: A longitudinal analysis. Learning Disability Quarterly, 29, 235-252.

Brucki, S. (2010). Illiteracy and dementia. Dementia \& Neuropsychologia, 4(3), 153-157.

Bryant, P., \& Bradley, L. (1987). Problemas de leitura na criança. Porto Alegre: Artes Médicas.

Carlisle, J. (1995). Morphological awareness and early reading achievement. In L. Feldman (Ed.), Morphological aspects of language processing (pp. 189-209). Hillsdale: Erlbaum.

Catts, H., Nielsen, D., Bridges, M., Liu, Y., \& Bontempo, D. (2015). Early identification of reading disabilities within an RTI framework. Journal of Learning Disabilities, 48, 281-297.

Catts, H., Petscher, Y., Schatschneider, C., Sittner, P., Bridges, M., \& Mendoza, K. (2009). Floor effects associated with universal screening and their impact on the early identification of reading disabilities. Journal of Learning Disabilities, 42, 163-176.

Clarke, P., Hulme, C., \& Snowling, M. (2005). Individual differences in RAN and reading: A response timing analysis. Journal of Research in Reading, 28, 73-86.

Cobbold, S., Passenger, T., \& Terrel, C. (2003). Serial naming speed and the component elements of speech time and pause time: Relationships with the development of word-level reading in children aged four to five years. Journal of Research in Reading, 26, 165-176.

Compton, D. L., Fuchs, D., Fuchs, L. S., \& Bryant, J. D. (2006). Selecting at-risk readers in first grade for early intervention: A two-year longitudinal study of decision rules and procedures. Journal of Educational Psychology, 98(2), 394-409.

Compton, D., Fuchs, L., Fuchs, B., Bouton, J., Gilbert, L., Barquero, E., ... Crouch, R. (2010). Selecting at-risk first-grade readers for early intervention: Eliminating false positives and exploring the promise of a two-stage gated screening process. Journal of Educational Psychology, 102(2), 327-340.

Denckla, M., \& Rudel, R. (1976). Rapid automatized naming (RAN): Dyslexia differentiated from other learning disabilities. Neuropsychology, 14, 471-479.

Di Filippo, G., Brizzolara, D., Chilosi, A., De Luca, M., Judica, A., Pecini, C., . . Z Zoccolotti, P. (2005). Rapid naming, not cancellation speed or articulation rate, predicts reading in an orthographically regular language (Italian). Child Neuropsychology, 11, 349-361.

Fletcher, J., \& Vaughn, S. (2009). Response to intervention: Preventing and remediating academic difficulties. Child Development Perspectives, 3(1), 30-37.

Georgiou, G., Parrila, R., Cui, Y., \& Papadopoulo, T. C. (2014). Why is rapid automatized naming related to reading? Journal of Experimental Child Psychology, 115, 218-225.

Hogan, T. (2006). Introdução à prática de testes psicológicos. Rio de Janeiro: LTC.

Jenkins, J., Hudson, R., \& Johnson, E. (2007). Screening for service delivery in an RTI framework: Candidate measures. School Psychology Review, 36, 582-599.
Johnston, A., Barnes, M., \& Desrochers, A. (2008). Reading comprehension: Developmental processes, individual differences, and interventions. Canadian Psychology, 49(2), 125-132.

Justi, C., \& Roazzi, A. (2012). A contribuição de variáveis cognitivas para a leitura e a escrita no português brasileiro. Psicologia: Reflexão e Crítica, 25(3), 400-409.

Justi, C., \& Roazzi, A. (2013). Nomeação seriada rápida e leitura: Quatro hipóteses sobre a relação entre essas variáveis. In A. Roazzi, F. Justi, \& J. Sales (Eds.), A aprendizagem da leitura e da escrita: Contribuições de pesquisas (pp. 109-130). São Paulo: Vetor.

Kirby, J., Pfeiffer. S., \& Parrila, R. (2003). Naming speed and phonological awareness as predictors of reading development. Journal of Educational Psychology, 95(3), 453-464.

Kruk, R., \& Funk, L. (2014). The predictive relations between nonalphanumeric rapid naming and growth in regular and irregular word decoding in at-risk readers. Journal of Research in Reading, 37(1), 17-35.

Landerl, K., \& Willbuger, E. (2010). Temporal processing, attention, and learning disorders. Learning and Individual Differences, 20, 393-401.

Lee, J., Chang, S., Jang, H., Chang, J., Suh, C., Jung, H., ... Cho, M. (2008). Illiteracy and the incidence of Alzheimer's disease in the Yonchon county survey, Korea. International Psychogeriatrics, 20(5), 976-985.

Lepola, J., Poskiparta, E., Laakkonen, E., \& Niemi, P. (2005). Development of and relationship between phonological and motivational processes and naming speed in predicting word recognition in grade 1. Scientific Studies of Reading, 9(4), 367-399.

Lervåg, A., Bråten, I., \& Hulme, C. (2009). The cognitive and linguistic foundations of early reading development: A Norwegian latent variable longitudinal study. Developmental Psychology, 45, 764-781.

Logan, J., Schatschneider, C., \& Wagner, R. (2011). Rapid serial naming and reading ability: The role of lexical access. Reading and Writing, 24, 1-25.

López-Escribano, C., \& Katzir, T. (2008). Are phonological processes separate from the processes underlying naming speed in a shallow orthography? Journal of Research in Educational Psychology, 16, 641-666.

Macklen, G. (2013). Prevention mental health at school: Evidencebased services for students. New York: Springer.

Norton, E. S., \& Wolf, M. (2012). Rapid automatized naming (RAN) and reading fluency: Implications for understanding and treatment of reading disabilities. Annual Review of Psychology, 63, 427-452.

O'Connor, R., \& Vadasy, P. (Eds.) (2011). Handbook of reading interventions. New York: The Guilford Press.

Parrila, R., Kirby, J., \& McQuarrie, L. (2004). Articulation rate, naming rapid speed, verbal short-term memory, and phonological awareness: Longitudinal predictors of early reading development? Scientific Studies of Reading, 8(1), 3-26.

Pauly, H., Linkersdörfer, J., \& Lindberg, S. (2011). Domain-specific rapid automatized naming deficits in children at risk for learning disabilities. Journal of Neurolinguistics, 24, 602-610. 
Pinheiro, A. (1996). Contagem de frequência de ocorrência e análise psicolinguística de palavras expostas a crianças na faixa pré-escolar e séries iniciais do $1^{\circ}$ grau. São Paulo: Associação Brasileira de Dislexia.

Pinheiro, A. (2007). Levantamento dos membros de categorias semânticas para itens verbais produzidos de forma escrita por crianças [Resumo]. In V. Golveia, C. Hutz, W. Lourenzo, \& E. Almeida (Eds.), III Congresso Brasileiro de Avaliação Psicológica e XII Conferência Internacional de Avaliação Psicológica: formas e contextos - Avaliação psicológica no século XXI: Ética e ciência-Livro de resumos/mesas redondas (pp. 34-35). João Pessoa: IBAP.

Plaza, M., \& Cohen, H. (2004). Predictive influence of phonological processing, morphological/syntactic skill, and naming speed on spelling performance. Brain and Cognition, 55, 368-373.

Puolakanaho, A., Ahonen, T., Aro, M., Eklund, K., Leppanen, P., Poikkeus, A., . . Lyytinen, H. (2007). Very early phonological and language skills: Estimating individual risk of reading disability. Journal of Child Psychology and Psychiatry, 48(9), 923-931.

Ritchey, K., \& Speece, D. (2004). Early identification of reading disabilities: Current status and new directions. Assessment for Effective Intervention, 29(4), 13-24.

Schatschneider, C., Fletcher, J., Francis, D., Carlson, C., \& Foorman, B. (2004). Kindergarten prediction of reading skills: A longitudinal comparative analysis. Journal of Educational Psychology, 96(2), 265-282.
Shapiro, L., Carroll, J., \& Solity, J. (2013). Separating the influences of prereading skills on early word and nonword reading. Journal of Experimental Child Psychology, 116, 278-295.

Snow, C., Burns, S., \& Griffin, P. (Eds.). (1998). Preventing reading difficulties in young children. Washington: National Academy Press.

Snowling, M., \& Hulme, C. (Eds.). (2005). The science of reading. Oxford, UK: Blackwell.

Stone, A., Siliman, E., Ehren, B., \& Apel, K. (Eds.). (2004). Handbook of language and literacy. New York: The Guilford Press.

Thompson, P., Hulme, C., Nash, H., Gooch, D., Hayiou-Thomas, E., \& Snowling, M. (2015). Developmental dyslexia: Predicting individual risk. Journal of Child Psychology and Psychiatry, 56(9), 976-987.

Torgesen, J. (1998). Catch them before they fall. Identification and assessment to prevent reading failure in young children. American Educator, 22(1), 32-39.

Verhagen, W., Aarnoutse, C., \& van Leeuwe, J. (2008). Phonological awareness and naming speed in the prediction of Dutch children's word recognition. Scientific Studies of Reading, 12, 301-324.

Wimmer, H., Mayringer, H., \& Landerl, K. (2000). The double deficit hypothesis and difficulties in learning to read a regular orthography. Journal of Educational Psychology, 92, 668-680.

Wolf, M., \& Denckla, M. (2005). RAN/RAS - Rapid automatized naming and rapid alternating stimulus tests: Examiner's manual. Texas: Pro-Ed.

Wolff, U. (2014). RAN as a predictor of reading skills, and vice versa: Results from a randomised reading intervention. Annual of Dyslexia, 64, 151-165.

Zhou, Y., McBride-Chang, C., Law, A., Li, T., Cheung, A., Wong, A., \& Shu, H. (2014). Development of reading-related skills in Chinese and English among Hong Kong Chinese children with and without dyslexia. Journal of Experimental Child Psychology, 122, 75-91. 